\title{
A Hierarchical A-Posteriori Error Estimator for the Reduced Basis Method
}

Stefan Hain*, Mario Ohlberger**, Mladjan Radic*, Karsten Urban*

*Ulm University, Institute for Numerical Mathematics

** University of Münster, Applied Mathematics

\section{Introduction}

We present an online-efficient hierarchical a-posteriori error estimator for the Reduced Basis Method (RBM), which uses the difference of two reduced approximations of different accuracy to estimate the true error of a reduced approximation. Effectivity as well as performance are investigated, especially for those cases where the inf-sup constant has small values and/or is hard to compute numerically.

\section{Reduced Basis Method (RBM)}

\section{Assumptions}

- $X, Y$ Hilbert spaces and $\mathscr{P} \subset \mathbb{R}^{P}$ parameter space,

- $a: \mathscr{P} \rightarrow\left[X^{\mathscr{N}} \times Y^{\mathscr{N}} \rightarrow \mathbb{C}\right]$ uniformly bounded and uniformly inf-sup stable sesquilinear form with inf-sup constant $\beta^{\mathcal{N}}(\mu)$, where $\mu \in \mathscr{P}$.

- $\beta_{\mathrm{LB}}^{\mathcal{N}}(\mu)$ computable lower bound of $\beta^{\mathcal{N}}(\mu)$.

- $f: \mathscr{P} \rightarrow\left[Y^{\mathscr{N}} \rightarrow \mathbb{C}\right]$ uniformly bounded linear form.

High-fidelity Problem

$X^{\mathscr{N}} \subset X, Y^{\mathscr{N}} \subset Y$ with $\operatorname{dim}\left(X^{\mathscr{N}}\right)=\operatorname{dim}\left(Y^{\mathscr{N}}\right)=\mathscr{N}<\infty$

- For $\mu \in \mathscr{P}$ find $u^{\mathscr{N}}(\mu) \in X^{\mathscr{N}}$, s.t.

$$
a\left(u^{\mathscr{N}}(\mu), v^{\mathscr{N}} ; \mu\right)=f\left(v^{\mathscr{N}} ; \mu\right) \quad \forall v^{\mathscr{N}} \in Y^{\mathscr{N}} .
$$

\section{Reduced Problem}

$X_{N} \subset X^{\mathscr{N}}$ RB space and $Y_{N} \subset Y^{\mathscr{N}}$ stabilized RB space with $\operatorname{dim}\left(X_{N}\right)=\operatorname{dim}\left(Y_{N}\right)=N \ll \mathscr{N}$

- For $\mu \in \mathscr{P}$ find $u_{N}(\mu) \in X_{N}$, s.t.

$$
a\left(u_{N}(\mu), v_{N} ; \mu\right)=f\left(v_{N} ; \mu\right) \quad \forall v_{N} \in Y_{N} .
$$

Residual Based Error Estimator (see e.g. $[7,8]$ )

$$
\left\|u^{\mathcal{N}}(\mu)-u_{N}(\mu)\right\|_{X} \leq \frac{\left\|R_{N}^{\mathcal{N}}(\cdot ; \mu)\right\|_{Y^{\prime}}}{\beta_{\mathrm{LB}}^{\mathcal{N}}(\mu)}=: \Delta_{N}^{\mathrm{Res}}(\mu),
$$

where $R_{N}^{\mathcal{N}}\left(v^{\mathscr{N}} ; \mu\right):=f\left(v^{\mathscr{N}} ; \mu\right)-a\left(u_{N}(\mu), v^{\mathscr{N}} ; \mu\right)$

\section{Saturation Property}

- $X_{N}$ and $X_{M}$ RB Spaces with $X_{N} \subsetneq X_{M} \subset X^{\mathcal{N}}$ Saturation property: If $\Theta_{N, M}^{\mathcal{N}} \in[0,1)$ exists, s.t.

$\left\|u^{\mathscr{N}}(\mu)-u_{M}(\mu)\right\|_{X} \leq \Theta_{N, M}^{\mathcal{N}}\left\|u^{\mathscr{N}}(\mu)-u_{N}(\mu)\right\|_{X} \quad \forall \mu \in \mathscr{P}$

\section{Outlook/Remarks}

- Offline approximation of $\Theta_{N, M}^{\mathcal{N}}$

- $\Delta_{N, M}(\mu)$ was applied to first order transport equations, see [3].

\section{References}

[1] R. E. Bank and R. K. Smith. A posteriori error estimates based on hierarchical bases. SIAM J. Numer. Anal., 30(4):921-935, 1993.

2] P. Binev, A. Cohen, W. Dahmen, R. DeVore, G. Petrova, and P. Wojtaszczyk. Convergence rates for greedy algorithms in reduced basis methods. SIAM J. Math. Anal., 43(3):1457-1472, 2011.

[3] J. Brunken, K. Smetana, and K. Urban. (Parametrized) First Order Transport Equations: Realization of Optimally Stable Petrov-Galerkin Methods. ArXiv e-prints, Mar. 2018

W. Dinkelbach. On nonlinear fractional programming. Management Science, 13(7):492-498, 1967.

Y. Huang, H. Wei, W. Yang, and N. Yi. A New a Posteriori Error Estimate for Adaptive Finite Element Methods, pages 63-74. Springer Berlin Heidelberg, Berlin, Heidelberg, 2011.

[6] D. B. P. Huynh, G. Rozza, S. Sen, and A. T. Patera. A successive constraint linear optimization method for lower bounds of parametric coercivity and inf-sup stability constants. C.R. Acad. Sci. Math., 345(8):473 - 478, 2007

A. Patera and G. Rozza. Reduced Basis Approximation and A Posteriori Error Estimation for Parametrized Partial Differential Equations. MIT, Cambridge (MA), USA, 2006. Version 1.0.

A. Quarteroni, A. Manzoni, and F. Negri. Reduced basis methods for partial differential equations: An introduction. Springer International Publishing, Cham; Heidelberg, 2016.

\section{A Hierarchical A-Posteriori Error Estimator}

\section{Preleminaries}

- For $X_{N} \subsetneq X_{M} \subset X^{\mathcal{N}}$ let $u_{N}(\mu) \in X_{N}, u_{M}(\mu) \in X_{M}$ solutions of (1) and define the hierarchical term

$$
\Delta_{\mathbf{N}, \mathbf{M}}(\mu):=\left\|u_{N}(\mu)-u_{M}(\mu)\right\|_{X} \cdot
$$

Asymptotic Approach

- The triangle inequality yields

$$
\left\|u^{\mathcal{N}}(\mu)-u_{N}(\mu)\right\|_{X} \leq\left\|u^{\mathcal{N}}(\mu)-u_{M}(\mu)\right\|_{X}+\left\|u_{M}(\mu)-u_{N}(\mu)\right\|_{X} \leq \Delta_{M}^{\mathrm{Res}}(\mu)+\Delta_{\mathbf{N}, \mathbf{M}}(\mu) .
$$

- If Kolmogorov M-width decays, $X_{M}$ may be constructed s.t. $\Delta_{M}^{\mathrm{Res}}(\mu) \leq \varepsilon \Delta_{\mathbf{N}, \mathbf{M}}(\mu)$ for all $\mu$, see [2]. Therefore

$$
\left\|u^{\mathcal{N}}(\mu)-u_{N}(\mu)\right\|_{X} \leq(1+\varepsilon) \Delta_{\mathbf{N}, \mathbf{M}}(\mu) .
$$

Approach by using the Saturation Property

- If the saturation property holds, it is well-knwon, see e.g. [1,5], that

$$
\frac{\Delta_{\mathbf{N}, \mathbf{M}}(\mu)}{1+\Theta_{N, M}^{\mathcal{N}}} \leq\left\|u^{\mathscr{N}}(\mu)-u_{N}(\mu)\right\|_{X} \leq \frac{\Delta_{\mathbf{N}, \mathbf{M}}(\mu)}{1-\Theta_{N, M}^{\mathcal{N}}}=: \Delta_{N, M}^{\mathrm{Hier}}(\mu) .
$$

- For the offline approximation of $\Theta_{N, M}^{\mathscr{N}}$ we suggest to decompose $\mathscr{P}$ in compact subsets $\mathscr{P}_{i}$ such that on each subset $\left\|u^{\mathcal{N}}(\mu)-u_{N}(\mu)\right\|_{X} \neq 0$ be valid. Solving the nonlinear problem, see [4]

$$
\Theta_{N, M, i}^{\mathcal{N}}:=\arg \min _{q \in \mathbb{R}_{\geq 0}}\left|F_{i}(q)\right| \quad \text { with } \quad F_{i}(q):=\max _{\mu \in \mathscr{P}_{i}}\left\{\left\|u^{\mathcal{N}}(\mu)-u_{M}(\mu)\right\|_{X}-q \cdot\left\|u^{\mathcal{N}}(\mu)-u_{N}(\mu)\right\|_{X}\right\}
$$

with a suitable initial value, we define $\Theta_{N, M}^{\mathcal{N}}:=\max _{i} \Theta_{N, M, i}^{\mathcal{N}}$

- For the RB space $X_{M}$ we suggest a Taylor-RB method, see e.g. [8], i.e.

$$
X_{M}:=\operatorname{span}\left\{u^{\mathscr{N}}\left(\mu_{n}\right), \frac{\partial^{k}}{\partial \mu_{i}^{k}} u^{\mathscr{N}}\left(\mu_{n}\right): k=1, \ldots, K_{n}, i=1, \ldots, P, n=1, \ldots, N\right\}, \quad K_{n} \in \mathbb{N}_{0} .
$$

- For the effectivity factor $\eta_{N, M}^{\mathcal{N}}(\mu):=\Delta_{N, M}^{\mathrm{Hier}}(\mu) /\left\|u^{\mathcal{N}}(\mu)-u_{N}(\mu)\right\|_{X}$ we get

$$
1 \leq \eta_{N, M}^{\mathcal{N}}(\mu) \leq \frac{1+\Theta_{N, M}^{\mathcal{N}}}{1-\Theta_{N, M}^{\mathcal{N}}}
$$

\section{Numerical Examples}

\section{Model Problem}

- Let $\mathscr{P}^{(1)}:=[95,100]$ and $\mathscr{P}^{(2)}:=[90,100]$.

- For $\mu \in \mathscr{P}^{(1)}$ or $\mu \in \mathscr{P}^{(2)}$ consider Helmholtz-Problem (1D)

$$
\begin{aligned}
-u^{\prime \prime}(x)-\mu^{2} u(x) & =1, \quad x \in(0,1), \\
u(0) & =0,
\end{aligned}
$$$$
u^{\prime}(1)+i \mu u(1)=0
$$

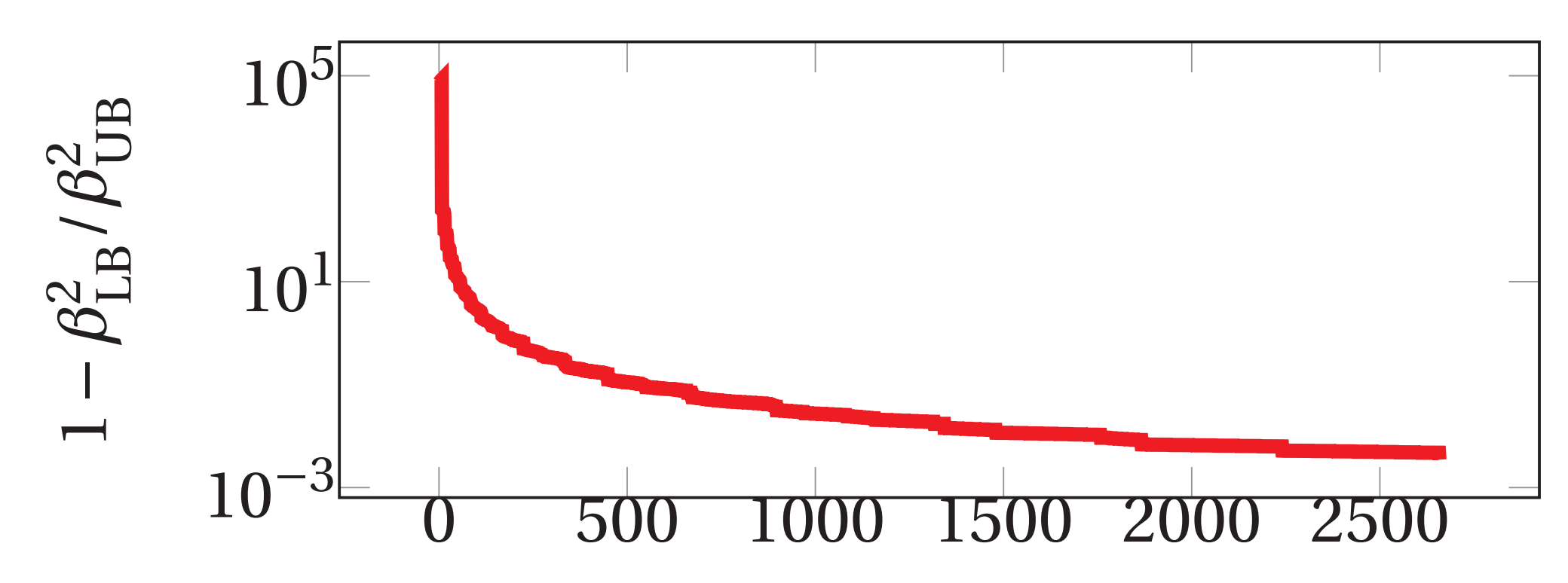

$N_{\mathrm{SCM}}$

Figure 1: SCM-convergence on $\mathscr{P}^{(1)}$

\section{Numerical Results - Error Plot for Model Problem}

- Parameter sampling with $\Delta_{\mathbf{N}, \mathbf{M}}(\mu)$

- Computation of $\beta^{\mathscr{N}}(\mu)$ with generalized eigenvalue problem.

- Computation of $\Theta_{N, M}^{\mathcal{N}}$ using $\sup _{\mu \in \mathscr{P}_{\text {train }}} \frac{\left\|u^{\mathcal{N}}(\mu)-u_{M}(\mu)\right\|_{X}}{\left\|u^{\mathcal{N}}(\mu)-u_{N}(\mu)\right\|_{X}}$.

- $\frac{\sum_{i=1}^{100}\left\|u^{\mathcal{N}}\left(\mu_{i}\right)-u_{N}\left(\mu_{i}\right)\right\|_{X}}{100}, \frac{\sum_{i=1}^{100} \Delta_{N}^{\mathrm{Std}}\left(\mu_{i}\right)}{100}$ and $\frac{\sum_{i=1}^{100} \Delta_{N, M}^{\mathrm{Hier}}\left(\mu_{i}\right)}{100}$ are shown in Figure 2 and 3, where $\mu_{1}, \ldots, \mu_{100}$ are randomly choosen.

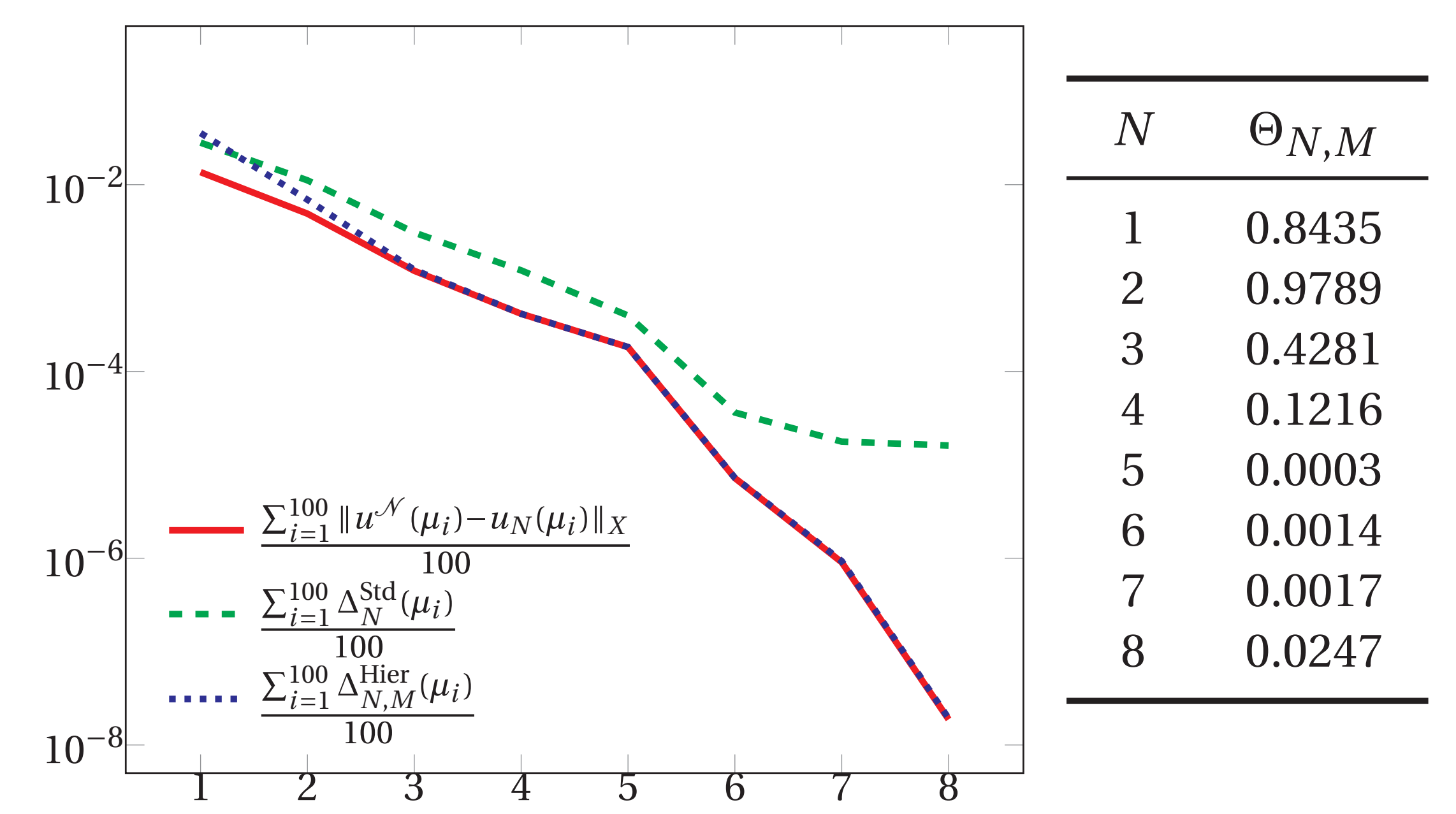

Figure 2: Parameter space $\mathscr{P}^{(1)}$ using $K_{n}=2$ for all $n \in \mathbb{N}$.

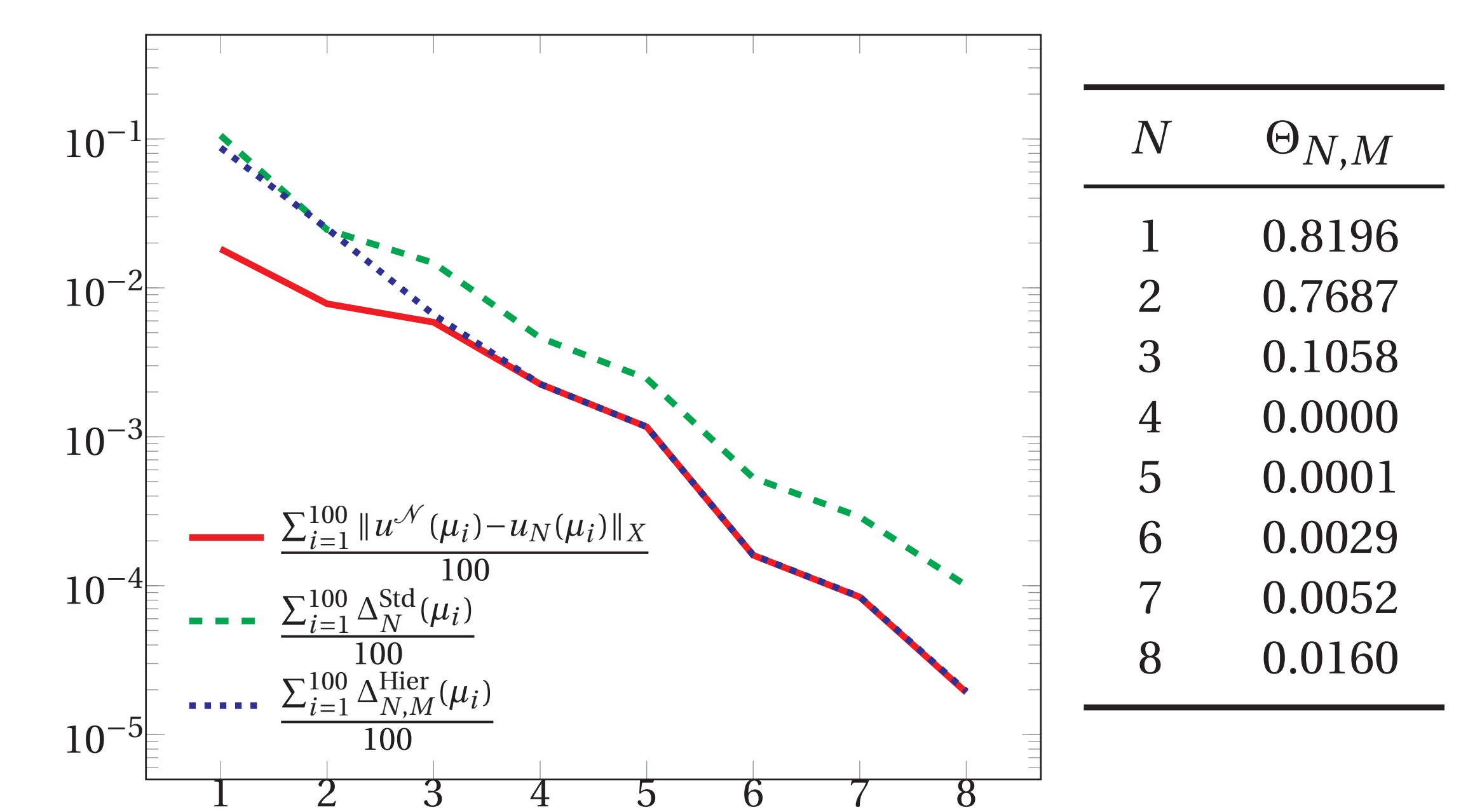

Figure 3: Parameter space $\mathscr{P}^{(2)}$ using $K_{n}=3$ for all $n \in \mathbb{N}$.
Numerical Results - Online CPU-Time for Model Problem

- $\beta_{\mathrm{LB}}^{\mathcal{N}}(\mu)$ computed with Successive Constraint Method (SCM), e.g. [6].

- SCM did not converge on $\mathscr{P}^{(2)}$. Therefore we consider only $\mathscr{P}^{(1)}$

- Lagrange-type basis construction with strong greedy for $X_{N}$ and $X_{M}$.

- $\eta^{\mathcal{N}}(\mu)$ controlled by SCM-tolerance, $\operatorname{dim}\left(X_{N}\right)$ and/or $\operatorname{dim}\left(X_{M}\right)$. Associated online CPU-time measured for randomly choosen parameter.

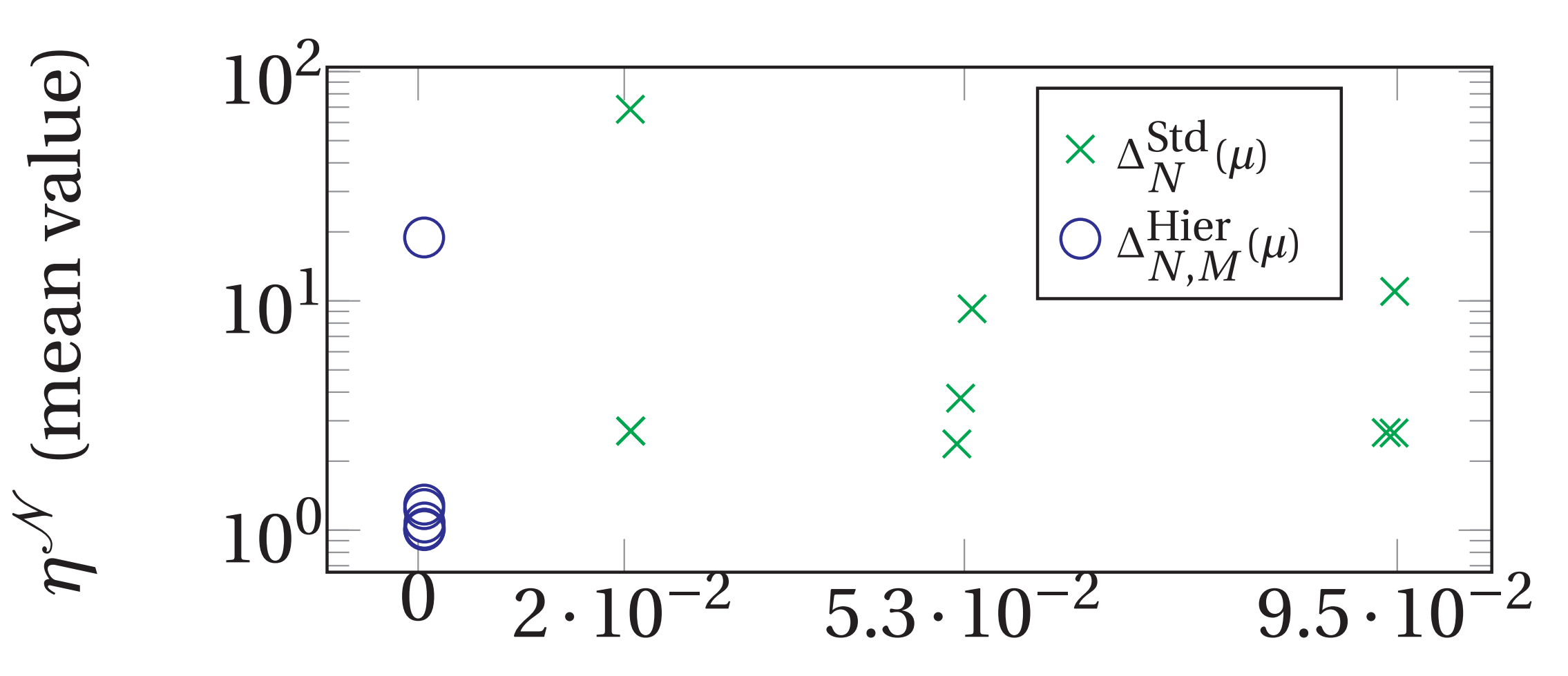

time $\cdot s^{-1}$ (mean value) 